\title{
Die Untersuchung und Beurteilung von Zitronensaft.
}

\author{
Von E. Fris c h - Hamburg.
}

(Eingegangen den 21. VII. 1908.)

Bis vor wenigen Jahren war der chemische Nachwcis selbst verhältnismäßig einfacher Zitronensaftverfälschungen ein oft aussichtsloses Unternehmen. Infolgedessen wurden diese Untersuchungen auf das Notwendigste beschränkt und vielfach sehr vernachlässigt. Hierdurch wuchs die Zahl der künstlichen Produkte, die infolge der mangelnden chemischen Kenntnisse über die Zusammensetzung des Zitronensaftea und der nicht ausreichenden Lntersuchungsmethoden unbeanstandet als unverfälschte Säfte ihren Weg zum Publikum fanden so erheblich, daß lange Zeit fast aller Zitronensaft. des Handels aus mehr oder minder geschickt zusammengesetzten Zitronensäurelösungen bestand. Erst durch die Arbeiten von

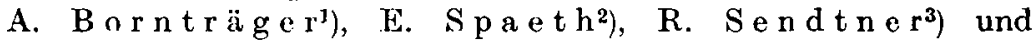
besonders von $\mathrm{K}$. Farnstein e $\mathrm{r}^{4}$ ) wurde es dem Chemiker ermöglicht, diesen Mißständen erfolgreich entgegenzutreten.

Unter Zitronensaft ist lediglich ein aus geschälten Zitronen durch Auspressen oder Zentrifugieren hergestellter Saft zu verstehen. Fr besteht nicht nur aus einer Lösung von Zitronensäure, sondern enthält außerdem noch Salze verschiedenster Art, Stickstoffsubstanzen, Bestandteile unbekannter Natur und Aromast offe. In ihrer Gesamtwirkung verleihen ihm diese seine Bedeutung als Genub- und Heilmittel. Nach $\$ 22$ der Anleitung zur Gesundheitspflege an Bord von Kuuffahrteischiffen, bearbeitet im Kaiserlichen Gesundheitsamte ${ }^{5}$ ), soll der vorschriftsmäßig mitzuführende Zitronensaft (lime or lemon juice), durch Auspressen von den Schalen befreiter Früchte gewonnen, ein natürliches, reines Produkt darstellen. Dasselbe darf nicht verdünnt werden, auch nicht den Zusatz irgend einer Säure enthalten; er muß von fleischigen Bestandteilen soweit befreit sein, daß sich beim Stehen kein Bodensatz mehr bildet.

Titronensaft aus gesunden Frïchten ist bei richtiger $\mathrm{Zu}$ bereitung und Behandlung jahrelang haltbar. Werden dagegen, wie es in Sizilien noch heute vielfach ïblich ist, abgefallene und verdorbene Früchte, die als solche zum Export untauglich sind,

1) Ztschr. f. Unters. d. Nahr.- u. Genußm. 1898, 1, 225-234.

2) Ztschr. f. Unters. d. Nahr.- u. Genußm. 1901, 4, 529-541.

3) Ztschr. f. Tinters. d. Nahr.- u. Genußm. 1901, 4, 1135-1140.

4) Ztschr. f. Unters. d. Nahr.- u. GenuBm. 1903, 1, 1-22.

3) Berlin, Julius Springer 1902. 
zur Herstellung von Saft verwandt, so kann natürlich nur ein minderwertiges Produkt von geringer Haltbarkeit entstehen. Dieser von dort exportierte Saft hat meist einen fauligen, unangenehmen Geruch und Geschmack, und eignet sich deshalb nicht für den häıslichen und medizinischen Gebrauch, scndern findet zu technischen Zwecken in Färbereien usw., sow ic auch zur Zitronensäurefabrikation Verwendung. Hensel und $\mathrm{Pr}$ in k e ${ }^{1}$, Zitronensaftpressereien in Görlitz i. Schl., Tschernhausen i. Böhm. und in Messina, machen über die Darstellung eines guten und haltbaren Zitronensaftes folgende Angaben: ",Die zur Zitronensaftbereitung benötigten Früchte sind reifer, feinster Sorte und werden sowohl mit der Hand, als auch mit Maschinen geschält, vermittelst Schneidemaschinen zerkleinert und entkernt. Darauf gelangen die Früchte in besonders hierfür konstruierte Pressen; der trübe Saft wird konserviert und geklärt. Die Klärung selbst erfolgt ohne jede chemische Hilfsmittel, der Versand in Glasballons oder in Fässern. Die Annahme, daß3 Zitronensaft in Faßbezügen leidet, bew ahrheitet sich nicht, da für diesen $\mathrm{Zweck}$ extra Fässer regelmäßig verwandt werden. Bezüglich Geschmack und Farbe ist ein solcher Saft nicht mit sogen. sizilianischen Exportzitronensaft zu verwechseln." A. B e ythien, J. Bohrisch und $\mathrm{H}$. Hem $\mathrm{pel}^{2}$ ) stellten ihre Zitronensäfte folgendermaßen her: „Die von Schale und Pulpe sorgfältig befreiten Früchte wurden kräftig ausgepreßt und die abfließenden Säfte in offenen tarierten Flaschen 8 Tage lang bei einer auch während der Nacht gleichmäßig auf $30^{\circ} \mathrm{C}$. erhaltenen Temperatur (Zimmertemperatur dürfte auch genügen) aufbewahrt. Nach Beendigung der lebhaft einsetzenden und unter starkem Schäumen verlaufenden Gärung wurden die Flüssigkeiten mit destilliertem Wasser zum ursprünglichen Gewicht ergänzt, durch Gaze kolicrt und mit 10\% Alkohol vermischt. Die einige Tage später nach dem Absitzen der Pektinstoffe ohne Anwendung von Klärungsmittein in der Kälte filtrierten Säfte erschienen völlig klar, besaßen prachtvolles Aroma und erhielten sich bis zum heutigen Tage (Mai 1906) völlig unverändert."

\section{Untersuohung des Zitrononsaftes.}

Alle Gewichtsangaben sind als Gramm in $100 \mathrm{ccm}$ auszudrücken.

Unter Zugrundelegung der neueren Literatur gestaltet sich die Zitronensaftuntersuchung folgendermaßen:

1) Pharm. Ztg. 1904, 7, 68.

2) Ztochr. f. Unters. d. Nahr.- u. Genußm. 1906, 11, 651-661. 
1. Spezifisches Gewicht des Saftes (S) wird im Pyknometer bestimmt.

2. Spezifisches Gewicht des entgeisteten Saftes $\left(S_{E}\right)$ : Nach Bestimmung des spezifischen Gewichtes des Saftes spült man den Pyknometerinhalt in eine Porzellanschale, dampft ihn auf dem Wasserbad auf $1 / 4$ des Volumens ein und füllt wieder in dasselbe Pyknometer bis zur Marke bei $15^{\circ} \mathrm{C}$. unter häufigem Umschütteln auf. Enthält der Saft flüchtige Säuren bezw. Aldehyde, so ist das Eindampfen nach vorhergehender Verdünnung mit Wasser noch mehrmals zu wiederholen und dann erst der entgeistete Saft in das Pyknometer überzuführen.

3. Alkohol: Durch Destillation. Bei Gegenwart flüchtiger Säuren ist der Saft vorher zu neutralisieren. Wenn wenig Material zur Verfügung steht, was bei den Zitronensaftuntersuchungen sehr oft vorkommt, genügt meist die Berechnung des Alkoholgehaltes aus den spezifischen Gewichten des Saftes und des entgeisteten Saftes $\left(1+S-S_{E}\right)$, natürlich aber nur dann, wenn die Abwesenheit flüchtiger Säuren zuivor festgestellt ist.

4. Zitronensäure: Diese Säure kommt in dem Zitronensaft frei, organisch und anorganisch gebunden vor.

a) Die freie $S$ ä ure ermittelt man durch Titration mit $1 / 2$ Normal-Lauge unter Benutzung von Phenolphthalein als Indikator. Es empfiehlt sich diese, wie überhaupt alle zur Extraktberechnung heranzuziehenden Bestimmungen doppelt auszuführen, da von ihrer Genauigkeit die wirkliche Größe des zur Beurteilung äußerst wichtigen Extraktrestes abhängt. Man verwendet $10 \mathrm{ccm}$ Saft (vorher abkühlen auf $15^{\circ}$ C.) und berechnet auf wasserfreie Zitronensäure. $\quad l \mathrm{ccm} \quad 1 / 2 \mathrm{~N} .-\mathrm{NaOH}=0,032 \mathrm{~g} \quad \mathrm{C}_{6} \mathrm{H}_{8} \mathrm{O}_{7} . \quad \mathrm{Un}$ verfälschte, besonders ältere Zitronensäfte färben sich beim Titrieren in der Nähe des Neutralisationspunktes oft derartig dunkel, daß letzterer schwierig oder überhaupt nicht zu erkennen ist. In solchem Falle muß der Saft mit Wasser so weit verdünnt werden, bis die dunkle Färbung nicht mehr störend wirkt. Die freie Säure ist auch in dem entgeisteten Saft zu bestimmen. Bei Abwesenheit von flüchtigen Säuren werden beide Werte übereinstimmen.

b) Organisch ge bundene, veresterte Zitronens ä u r e: Man neutralisiert genau $10 \mathrm{ccm}$ Saft, gibt weiter $10 \mathrm{ccm}$ $1 / 2$ N.-NaOH hinzu, versehliebt das Kölbchen mit Korkstopfen und läßt es 2 Stunden im Dunkeln stehen. Darauf titriert man mit $1 / 2$ Normal-Salzsäure mit Phenolphthalein als Indikator zurück. Hier macht sich die oben besprochene Dunkelfärbung in erhöhtem Maße bemerkbar, so daß unter Umständen ein recht erheblicher 
Wasserzusatz notwendig werden kann. Bei hohem Gehalt an veresterter Zitronensäure kann ein Zusatz von $20 \mathrm{ccm} \frac{1}{2}$-Normal$\mathrm{NaOH}$ notwendig sein. Die Berechnung erfolgt als wasserfreie Zitronensäure wie bei a.

c) Anorganisch gebundene Zitronensäure wird aus der Alkalität der Mineralstoffe berechnet, siehe Extrakt.

5. Flüchtige Säuren: Unverfälschter und unverdorbener Zitronensaft enthält keine oder nur schr geringe Mengen (Spuren) flüchtiger Säuren. Werden nun in einem Saft größere Mengen flüchtiger Säuren gefunden, die nicht in Gestalt von Ameisensäure absichtlich zur Konservierung zugesetzt sind, so wird meist der Gehalt an nichtflüchtigen Säuren niedrig sein. Denn wie bekannt, wird die Zitronensäure unter der Einwirkung gewisser Spaltpilze zerstört und durch eine besondere Gärung in Essigsäure übergeführt. Außer flüchtigen Süuren können sich hierbei, wie K. Farnste in e $\mathbf{r}^{1}$ ) gezeigt hat, auch neutrale flüchtige, reduzierende Körper (Aldehyde und ähnliche Verbindungen) bilden. Bei Gegenwart dieser Stoffe muß man den Zucker in dem entgeisteten Saft bestimmen (ein wiederholtes Eindampfen ist hierbei zu empfehlen), da man sonst selbst bei unverfälschtem Saft leicht zu negativen Extraktresten kommen kann; denn diese die F e hl in g'sche Lösung reduzierenden Körper können einen recht erheblichen Zuckergehalt vortäuschen, wie folgende von F a r ns t e in er angegebene Beispiele ergeben:

\begin{tabular}{|c|c|c|c|}
\hline & \multicolumn{3}{|c|}{ Gramm in $100 \mathrm{ccm}$} \\
\hline & I & II & III \\
\hline Alkohol . & - & 0,58 & 0,74 \\
\hline Extrakt & 6,60 & 6,11 & 6,82 \\
\hline Mineralstoffe & 0,55 & 0,41 & 0,43 \\
\hline Nichtflüchtige Säuren & $\left.3,26^{1}\right)$ & $\left.4,26^{1}\right)$ & $\left.3,61^{1}\right)$ \\
\hline Flüchtige Säuren . . & 0,20 & $\begin{array}{l}\left.0,29^{2}\right) \\
\left.0,87^{3}\right)\end{array}$ & $\left.1,48^{4}\right)$ \\
\hline yd durch das Destillat & 0,214 & 1,776 & 1,398 \\
\hline Scheinbarer Zuckergehalt des Destillates & 0,09 & 0,75 & 0,57 \\
\hline „Aldehyd" aus dem Kupferoxyd berechnet & 0,06 & 0,49 & 0,38 \\
\hline 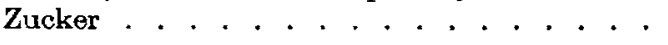 & 1,87 & 0,63 & 2,03 \\
\hline
\end{tabular}

1) Zitronensäure. $-{ }^{2}$ ) Ameisensäure. $-{ }^{3}$ ) Essigsäure. $\left.-{ }^{4}\right)$ Einschließlich Ameisensäure.

I) Ztachr. f. Unters. d. Nahr.- u. Genußm. 1908, 15, 323. 
6. Zucker: Natürlicher Zitronensaft enthält nur geringe Mengen, meist unter $1-2 \mathrm{~g}$ Zucker in $100 \mathrm{ccm}$ Saft, und zwar Invertzucker. Der Saft des Handels erfährt oft einen Zuckerzusatz (Rohrzucker). Dieser wird durch die Säure des Saftes beim Lagern invertiert. Die Inversion dürite bei einem $1 \mathrm{Jahr}$ alten Saft in den meisten Fällen größtenteils beendet sein. Wenn keine besondere Veranlassung vorliegt, wird die Zuckerbestimmung nur in invertierten Saft ausgeführt. Zur Inversion erhitzt man eine abgemessene Menge Saft olne weiteren Säurezusatz in einem Kölbchen $1 / 2$ Stunde im kochenden Wasserbad. Nach Ablauf dieser Zeit ist sicher etwa noch vorhanden gewesener Rohrzucker in Invertzucker übergeführt. Nach dem Abkühlen neutralisiert man und fiillt anf das gewünschte Volumen auf.

7. Mineralstoffe und Alkalität der Mineralstoffe werden aus $25 \mathrm{ccm}$ unter Beachtung der Vorsichtsmaßregeln bestimmt, welche $\mathrm{P}$. Butte $\mathrm{n} \mathrm{ber}_{\mathrm{r}}{ }^{1}$ ) in seiner Arbeit ,Die Untersuchung und Beurteilung des Himbeersaftes und Himbeersirupes" eingehend beschrieben hat. Noch genauere Werte, als nach dem bisherigen Verfahren zur Bestimmung der Alkalität der Mineralstoffe, erhält man mit dem Farnsteiner'schen Fällungsverfahre $\left.{ }^{2}\right)$ : „Hierbei ist ebenfalls auf eine sorgfältige Darstellung der Asche besonderer Wert zu legen. 0,2-0,3 g der scharf getrockneten Asche rührt man mit etwas Wasser zu einem feinen Brei an und bringt dieselbe in bedecktem Gefäß mit $10-20 \mathrm{ccm}$ 1/2 Normal-Salzsäure (genau abgemessen) bei gelinder Wärme in Lösung. Die saure Lösung bringt man mit Hilfe von $30-40 \mathrm{ccm}$ Wasser verlustlos in ein Erlenmeyer-Kölbchen von etwa $150 \mathrm{ccm}$ Inhalt, erhitzt die Flüssigkeit zum Sieden und läßt sie bei ganz klein gestellter Flamme unter mehrfachem Umschwenken etwa 3-5 Minuten kochen. Hierauf kühlt man ab und führt die kalte Lösung mit 20-30 ccm Wasser in einen mit Glasstopfen verschließbaren Meßzylinder über. $\mathrm{Zu}$ der nach Möglichkeit gemischten Flüssigkeit werden dann 5-10 ccm streng neutraler Chlorcalciumlösung $(5 \mathrm{~g}$ trockenes Chlorcalcium und $10 \mathrm{~g}$ Chlorammonium zu $100 \mathrm{ccm}$ ) und $10-20 \mathrm{ccm}$ einer etwa halbnormalen Ammoniaklösung hinzugesetzt und das Volumen mit kohlensäurefreiem Wasser auf $100 \mathrm{ccm}$ gebracht. Nach mehrfachem kräftigem Umschütteln des gut verschlossenen Zylinders läßt man die Flüssigkeit über Nacht zum Absetzen des Niederschlages stehen. Schließlich werden

1) Arch. d. Pharm. 1907, 245, 81-97.

2) Ztschr. f. Unters. d. Nahr.- u. Genußm. 1907, 13, 305-338. 
25-50 ccm der klaren Flüssigkeit mit der Pipette entnommen und nach Zusatz einiger Tropfen Methylorange mit 1/10 Normal-Salzsäure titriert. Bezeichnet

a das Gewicht der Asche in Gramm,

$S$ das Volumen der zur Lösung verwendeten Säure in Kubikzentimeter Normal-Säure,

$\mathrm{n}$ das Volumen des zugesetzten Ammoniaks in Kubikzentimeter Normal-Ammoniak,

s das Volumen der beim Zurücktitrieren für die ganze Substanzmenge verbrauchten Säure in Kubikzentimeter Normal-Säure,

so ist die Alkalität für a Gramm Asche: $a=S+s-n . "$

8. Stickstolf nach Kjeldahl: Aus $50 \mathrm{ccm}$ Saft. Es empfiehlt sich, den Saft im Stickstoffkolben im Wasserbad bis zur Sirupdicke einzudampfen, um beim Aufschließen zu starkes Schäumen zu vermeiden.

9. Glyzerin: Aus $50 \mathrm{ccm}$ Saft nach der amtlichen. Weinvorschrift a mit entsprechend größerem Kalkzusatz. Man bringt die alkoholische Flüssigkeit statt auf 100 auf $200 \mathrm{ccm}$.

Da das zur Wägung gelangte Glyzerin nicht rein ist, empfiehlt es sich, wie bislang unveröffentlichte Versuche im Hygienischen Institut zu Hamburg ergeben haben, den Gehalt an Reinglyzerin analytisch festzustellen und nur diesen Wert bei der Extraktbestimmung zu berücksichtigen. Von den vielen hierzu geeigneten Methoden möchte ich das Oxydationsverfahren mit Kaliumpermanganat in alkalischer Lösung von Benedikt und $\mathrm{Z}$ sig m o n d $\mathrm{y}^{\mathbf{1}}$ ), herausgreifen. Bei diesem Verfahren findet folgende Umsetzung statt:

$$
\begin{aligned}
& \stackrel{\mathrm{CH}_{2} \mathrm{OH}}{\mathrm{CH}} . \mathrm{OH} \\
& \underset{\mathrm{CH}}{\mathrm{O}} \mathrm{OH}
\end{aligned}
$$

$0,2-0,5 \mathrm{~g}$ Rohglyzerin in wässeriger Lösung werden in einem Erlenmeyerkolben von etwa $300 \mathrm{ccm}$ Inhalt (nicht kleiner !) mit Wasser auf etwa $150 \mathrm{ccm}$ gebracht und $10 \mathrm{~g}$ festes Aetzkali darin aufgelöst. $\mathrm{Zu}$ der abgekühlten Lösung gibt man etwa $40 \mathrm{ccm} 5 \%$ ige Kaliumpermanganatlösung bis zur Blaugrünfärbung, läßt 15 Minuten stehen, erhitzt und läßt einmal aufkochen. Die heiße Lösung versetzt man mit 10\% \%ger Natriumsulfitlösung bis zum Farben-

1) Chem.:Ztg. 1885, 9, 975 und B e n edikt-Ulzer, Chemie d. Fette u. Wachsarten, Berlin, Julius Springer 1908, 5. Aufl., S. 196. 
umschlag in Braun (etwa $15 \mathrm{ccm}$ ) und bis beim Stehenlassen die überstehende Flüssigkeit farblos ist. Ein Ueberschuß von $\mathrm{Na}_{2} \mathrm{SO}_{3}$ Lösung ist $\mathrm{zu}$ vermeiden. Man füllt dann auf $250 \mathrm{ccm}$ auf und filtriert durch ein glattes Filter, das mindestens die Hälfte der ganzen Flüssigkeit auf einmal aufnehmen kann. $200 \mathrm{ccm}$ Filtrat werden mit Essigsäure angesäuert und mit Chlorcalciumlösung in der Siedehitze gefällt. Der erhaltene Niederschlag wird nach hinreichendem Stehenlassen filtriert, ausgewaschen, bis zur Gewichtskonstanz geglüht und als $\mathrm{CaO}$ gewogen. 1 Gewichtsteil $\mathrm{CaO}=$ 1,643 Gewichtsteile Glyzerin. Ein Ueberschuß von $\mathrm{Na}_{2} \mathrm{SO}_{3}$-Lösung ist deshalb zu vermeiden, weil der Niederschlag sonst leicht durch mitausgefälltes $\mathrm{CaSO}_{4}$ verunreinigt wird. Man umgeht diesen Uebelstand ganz, nach bislang noch unveröffentlichton Untersuchungen von $K$. F a r n s t e i n e r, durch Verwendung von etwa $10 \mathrm{ccm} 1 \%$ iger Formalinlösung an Stelle der $\mathrm{Na}_{2} \mathrm{SO}_{3}$-Lösung.

10. Die Untersuchung auf künstliche Färbung, künstliche Süßstoffe und Konservierungsmittel ist in gleicher Weise auszuführen wie bei Himbecrsäften. (Siehe die oben genannte Arbeit von P. But t en berg.)

\section{Qualitative Reaktionen:}

a) mit Natronlauge: Man versetzt einige Kubikzentimeter Saft nach und nach mit verdünnter Natronlauge bis zur alkalischen Reaktion. Dunkelfärbung (braungelb bis dunkelbraun) gilt als Charakteristikum von reinen Säften. Doch wurden verschiedentlich reine Säfte beobachtet, die eine Dunkelfärbung. nicht gaben.

b) mit A m mo niak: analog wie a.

c) mit A l k o h o l: Man versetzt einige Kubikzentimeter Zitronensaft mit der etwa dreifachen Menge Alkohol. Bei reinen Säften tritt eine weiße Trübung, unter Umständen ein weißer Niederschlag nach längerem Stehen auf.

Die Reaktionen a, b und c geben dem Analytiker wichtige Anhaltspunkte zur Beurteilung, doch kann nicht aus dem Ausbleiben der einen oder anderen Reaktion auf eine Verfälschung des Saftes geschlossen werden; sie helfen nur das Gesamtbild vervollständigen.

12. Extrakt ${ }^{1}$ ): Da eine Extraktbestimmung durch Wägung keine vollständig einwandfreien Ergebnisse liefert, muß man sich

1) Ztschr. f. Unters. d. Nahr.- u. Genußm. 1903, 1, 1-22. Ztschr. f. Unters. d. Nahr.- u. Genußm. 1904; 8, 593-603. F. L e p è r e, Ztschr. öff. Chem. 1906, 12, $1-10$. 
der Farnsteiner'schen Extraktbestimnung, des sogenannten Additionsverfahren $\mathbf{s}^{1}$ ), bedienen. Es beruht auf der Addition der Werte von folgenden Extraktbestandteilen: Zitronensäure, Zucker, Mineralstoffe und daran gebundene Zitronensäure, Glyzerin und Extraktrest. Während Zitronensäure, Zucker, Mineralstoffe und daran gebundene Zitronensäure sowie Glyzerin analytisch gefunden werden, wird der Extraktrest berechnet, wozu die spezifischen Gewichte des entgeisteten Saftes und der oben genannten Extraktbestandteile benutzt werden. Um nicht immer mit den fünf- und mehrstelligen Zahlen der spezifischen Gewichte rechnen zu müssen, ist der Begriff a eingeführt. Dieser bezeichnet die Zahl, die angibt, wieviel Milligramme $1 \mathrm{ccm}$ mehr wiegt wie $1 \mathrm{~g}$. Zum Beispiel eine wässerige Zitronensäure-

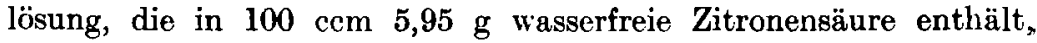
hat ein spezifisches Gewicht von 1,02508 oder $a_{c}=25,08$.

Der dem spezifischen Gewicht des entgeisteten Saftes entsprechende Wert $a_{E}$ setzt sich aus den Werten von a für die einzelnen Bestandteile zusammen. Den Wert für Zitronensäure - $a_{a}-$ findet man aus der Tabelle, die von Prof. Dr. K. F a rnst e in er neu aufgestellt und mir zur vorliegenden Veröffentlichung überlassen worden ist.

Zur Ermittelung des Wertes von a für Zucker $-a_{z}-$ ist die amtliche Zuckertabelle anzuwenden. In frischen gezuckerten Zitronensäften ist oft noch Rohrzucker vorhanden. Beim Erhitzen, wie es zur Ermittelung des spezifischen Gewichtes des entgeisteten Saftes notwendig ist, tritt Inversion ein, so daß der entgeistete Saft praktisch keinen Rohrzucker mehr enthält. Deshalb ist die Erhöhung, die das spezifische Gewicht einer Rohrzuckerlösung durch Inversion erfährt, nicht weiter zu berücksichtigen. Die amtliche Rohrzuckertabelle hat auch für Invertzucker Gültigkeit.

Der oben gefundene Wert für $a_{c}$ faßt nur die freie und die organisch an Alkohol gebundene Zitronensäure zusammen. Die anorganisch gebundene Zitronensäure finden wir als Kohlensäure in der Asche wieder. Die Gewichtsveränderung, welche die Asche infolge des Ersatzes von $\mathrm{CO}_{2}$ durch $\mathrm{C}_{6} \mathrm{H}_{8} \mathrm{O}_{7}$ erfährt, festzustellen, gestattet folgende Gleichung:

$$
3 \mathrm{~K}_{2} \mathrm{CO}_{3}+2 \mathrm{C}_{6} \mathrm{H}_{8} \mathrm{O}_{7}=2 \mathrm{~K}_{3} \mathrm{C}_{6} \mathrm{H}_{5} \mathrm{O}_{7}+3 \mathrm{CO}_{2}+3 \mathrm{H}_{2} \mathrm{O} \text {, }
$$

1) Ztschr. f. Unters. der Nahr.- u. Genußm. 1903, 1, 1-22. Ztschr. f. Untere. d. Nahr.- u. GeouBm. 1904, 8, 593-603. E. L e p èr e, Ztsohr. öff. Chern. 1906, 19, $1-10$. 
wobei man von der Ueberlegung ausgeht, daß die Zitronensäure anorganiseh an Kalium gebunden ist. Es entsprechen : $3 \mathrm{~K}_{2} \mathrm{CO}_{3}=$ $2 \mathrm{~K}_{3} \mathrm{C}_{6} \mathrm{H}_{5} \mathrm{O}_{7}$.

Man erhält, wenn man von $2 \mathrm{Mol} . \mathrm{K}_{3} \mathrm{C}_{6} \mathrm{H}_{5} \mathrm{O}_{7}$ oder $\mathrm{K}_{6} \mathrm{C}_{12} \mathrm{H}_{10} \mathrm{O}_{14}$ $3 \mathrm{Mol} . \mathrm{K}_{2} \mathrm{CO}_{3}$ oder $\mathrm{K}_{6} \mathrm{C}_{3} \mathrm{O}_{9}$ abzieht, eine Gewichtsändêrung von 198. Mithin liefert 1 Aequivalent $\mathrm{K}_{2} \mathrm{CO}_{3}$ oder vielmehr 1 Aequivalent $\mathrm{CO}_{2}$ einen Gewichtsüberschuß von $\frac{198}{6}=33$. Den durch Einführung von $\mathrm{C}_{6} \mathrm{H}_{8} \mathrm{O}_{7}$ entstehenden Gewichtsüberschuß findet man, wenn man die zur Neutralisation verbrauchten Kubikzentimeter NormalSäure mit 0,033 multipliziert. Addiert man dieses Produkt (Alkalität mal 0,033) und die Mineralstoffe, so hat man den Wert für die Mineralstoffe und die an diese gebundene Zitronensäure gefunden. Der Wert a für $I \mathrm{~g}$ (Mineralstoffe - anorganisch gebundene Zitronensäure) in $100 \mathrm{~cm}-\mathrm{m}+\mathrm{c}-$ ist gleich 7,0 . Hat man.z. B. für $\mathrm{m}+\mathrm{c}=0,56$ gefunden, so ist $\mathrm{a}_{\mathrm{ul}}+\mathrm{c}=0,56 \mathrm{mal} 7,0=3,92$.

In vielen Fällen hat es sich als notwendig erwiesen, buch das Glyzerin bei diesen Berechnungen heranzuziehen, weil künstlichen Produkten häufig Glyzerin zugesetzt wird. Da $1 \mathrm{~g}$ Glyzerin in wässeriger Lösung in $100 \mathrm{ccm}$ ein spezifisches Gewicht von 1,00239 hat, oder $a_{\text {(ilyz. }}=2,39$ ist, muß der gefundene Wert für Glyzerin mit dem Faktor 2,39 multipliziert werden.

Den totalen Extraktrest bezeichnet man mit e. $a_{4}=a_{F}$ $-\left(a_{w}+a_{z}+a_{m}+c+a_{(i l y \%}\right) ; \quad F=$ entgeisteter Saft. $a_{k}$ kann meist nicht ohne weiteres zur Berechnung herangezogen werden, da fast alle Zitronensäfte Ester enthalten. Ebenso wie in den Estern die Zitronensäure berechnet wird, muß in ihnen auch der Alkohol Berücksichtigung finden. $K$. F a r n s t e i n e r nimmt an, daß bei den niedrigen Konzentrationen, wie sie hier in Betracht kommen, eine Lösung des Zitronensäureäthylesters $\mathrm{C}_{6} \mathrm{H}_{5} \mathrm{O}_{7}\left(\mathrm{C}_{2} \mathrm{H}_{5}\right)_{3}$ dasselbe spezifische Gewicht hat, wie ein entsprechendes Gemisch von Süure und Alkohol. Man muß nur den gebundenen Alkohol ermitteln und das auf denselben in der betreffenden Konzentration entfallende spezifische Gewicht berücksichtigen. Da der Ester bei der Verseifung in $\mathrm{C}_{6} \mathrm{H}_{8} \mathrm{O}_{7}$ und $3 \mathrm{C}_{2} \mathrm{H}_{5} \mathrm{OH}$ zerfällt, entspricht 1 Gewichtsteil gebundener Zitronensäure 0,72 Gewichtsteilen Alkohol. Man hat also nur den für die Estersäure berechneten Wert mit 0,72 zu multiplizieren und für das gefundene Resultat das spezifische Gewicht in der Alkoholtabelle aufzuschlagen. Das korrigierte spezifische Gewicht des entgeisteten Saftes $S_{F}$ ist dann gleich $1+S_{\mathrm{E}}$ - Sa. Sa sei das spezifische Gewicht des Esteralkohols.

(Fortsetzung folgt.) 
Addiert man die für a gefundenen Zalken von $c, z, m+c$ und Glyzerin und zieht diese Summe von $a_{\varepsilon} a b$, so erhält man $a_{\circ}$ und findet in der Zuokertabelke aus diesem spezifischen Gewicht des Extraktrestes seinen eigentlichen Wert. Ein Beispiel mag dies näher veranschaulichen:

$$
\begin{aligned}
& \text { Gramm in } 100 \mathrm{ccm}
\end{aligned}
$$

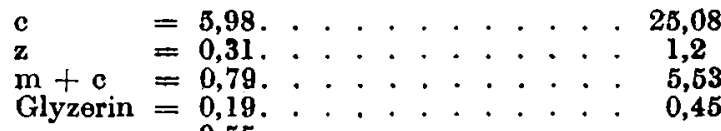

$$
\begin{aligned}
& \frac{\theta}{\text { Extrakt }}=7,8,52 \mathrm{~g} \text { in } 100 \mathrm{ccm} \text {. }
\end{aligned}
$$

Hiernach ist der Extrakt des Zitronensaftes gleich der Summe der Werte für Zitronensäure, Zucker, Mineralstoffe und an diese gebundene Zitronensäure, Glyzerin und Extraktrest, in diesem Falle also gleich 7,82 .

Tabellezur Ermittelung des Wertes a.

\begin{tabular}{|c|c|c|c|c|c|c|c|c|c|c|}
\hline \multirow{2}{*}{ 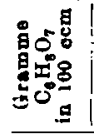 } & \multicolumn{10}{|c|}{ Zehntelgramme } \\
\hline & 0,0 & 0,1 & 0,2 & 0,3 & 0,4 & 0,5 & 0,6 & 0,7 & 0,8 & $0, \theta$ \\
\hline 1 & 4,22 & 4,64 & 5,06 & 5,48 & $5, \theta 1$ & 6,33 & 6,75 & 6,11 & 7,59 & 8,02 \\
\hline 2 & 8,44 & 8,86 & 9,27 & 9,69 & 10,11 & 10,53 & 10,95 & 11,37 & 11,79 & 12,21 \\
\hline 3 & 12,63 & 13,05 & 13,46 & 13,88 & 14,30 & 14.72 & 15,14 & 15,56 & 15,98 & 16,40 \\
\hline 4 & 16,82 & 17,23 & 17,65 & 18,07 & 18,49 & 18,91 & 19,32 & 19,74 & 20,16 & 20,57 \\
\hline $\mathbf{5}$ & 20,99 & 21,41 & 21,82 & 22,24 & 22,66 & 23,07 & 23,49 & 23,91 & 24,32 & 24,74 \\
\hline 6 & 25,16 & 25,57 & 25,99 & 26,41 & 26,83 & 27,24 & 27,66 & 28,07 & 28,49 & 28,90 \\
\hline 7 & 29,32 & & & 30,56 & 30,97 & 31,39 & 31,80 & 32,22 & 32,63 & 33,05 \\
\hline 8 & 33,46 & 33,88 & 34,29 & 34,70 & 35,12 & 35,53 & 35,94 & 36,35 & 36,77 & 37,18 \\
\hline 9 & 37,59 & 38,01 & 38,42 & 38,83 & 39,25 & 39,66 & 40 & 9 & 40 & 41,31 \\
\hline 10 & 41,72 & 42,13 & 42,54 & 42,95 & 43,37 & 43,78 & 44,19 & 44,60 & 46,01 & 45,42 \\
\hline 11 & 45,84 & 46,25 & 46,66 & 47,06 & 47,47 & 47,88 & 48,29 & 48,70 & 48,11 & 49,52 \\
\hline 12 & 49,93 & 50,34 & 50,75 & 51,16 & 61,57 & 51,98 & 52,39 & 62,80 & 58,21 & 53,62 \\
\hline 13 & 54,03 & 54,44 & 54,85 & 55,26 & 55,66 & 56,07 & 56,48 & 56,88 & 57,30 & 57,71 \\
\hline 14 & $|58,11|$ & 58,52 & 58,93 & - & - & - & - & 一 & - & $\longrightarrow$ \\
\hline
\end{tabular}

\section{Bourtellung des Zitronensaftes.}

Zitronensaft besitzt frisch gepreßt eine grünlich-gelbe Farbe, die bcim Lagern nachdunkelt und mehr und mehr ins Bräunliche hinüberspielt. Sein eigenartiges Fruchtaroma hat fast keinerlei Achnlichkeit mit dem Aroma der Schalen; auch der Geschmack ist charakteristiseh und kann bei einiger Uebung nicht mit dem von Zitronensäurelösungen verwechselt werden. 
Der Gehalt an freier Zitronensëure schwankt zwischen $5,0-8,0$ g $\mathrm{C}_{6} \mathrm{H}_{8} \mathrm{O}_{7}$ in 100 com Saft. Der Höchstwert ist hierbei sehr hoch angenammen Dagegen können in seltenen Fällen auch geringere Werte wie 5,0 erhalten werden: $z$. B.. hat $A$. $B$ e y $t h$ i e $n^{1}$ ) Säfte mit 4,3-5,0 g Zitronensäure untersucht. In den vergorenen und gespriteten Säften bilden sich nach kurzer Zeit ZitronensëureEster, die beim Lagern allmählich zunehmen und unter normalen Verhältnissen einen Wert bis $0,5 \mathrm{~g}$, auf Zitronensäure berechnet, erreichen können; in den meisten Fällen findet man Werte um $0,1 \mathrm{~g}$ als $\mathrm{C}_{6} \mathrm{H}_{8} \mathrm{O}_{7}$ berechnet. Die Esterbildung beginnt, wie A. Beythien festgestellt hat, bei energischem Verlauf der Gärung und nachfolgeadem Alkoholzusatz schon innerhalb kurzer Zeit:

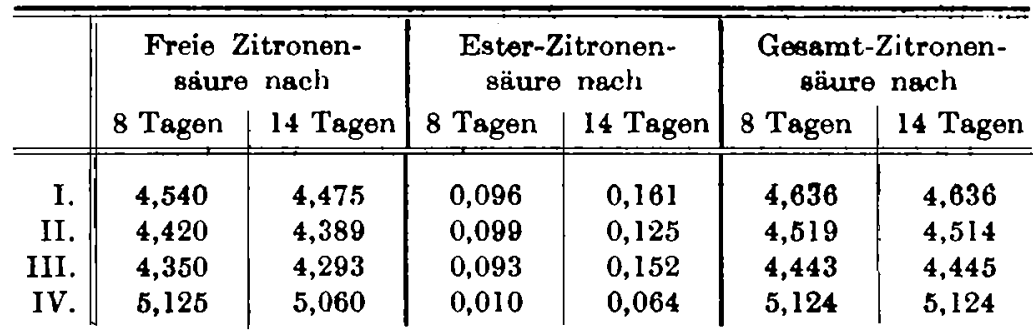

In Uebereinstimmung mit A. B ornträger, E. S p a e th und R. Sendtner fand $K$. Farnsteine $r 0,38-0,59 \mathrm{~g}$ Mineralstoffe in $100 \mathrm{ccm}$ Saft; ähnliche Werte wurden auch von anderen Analytikern gefunden. Während $\left.F . \mathrm{Lü}^{\mathrm{h}} \mathbf{r} \mathrm{i}^{1}{ }^{1}\right)$ in seinen Angaben bis auf $0,30 \mathrm{~g}$ herabgeht.

Die Alkalität der Mineralstoffe steigt von etwa 4,5 bis annähernd $8,0 \mathrm{ccm} 1 / 1$ Normal-Säure.

Auch der Stickstoffgehalt ist beträchtlichen Schwankungen unterworfen, Es scbeint, daB die Herstellungsart der Säfte von ziembichem Einfluß auf ihn ist, wenn such nicht in dem Maße, wie von den Fabrikanten der Kunstprodukte des öfteren angegeben wird. So wurden folgende Werte gefunden:

Gramm in $100 \mathrm{ccm}$

K. Farnsteiner... $0,055-0,093$

A. Beythien . . . . 0,038-0,067 1804er Säfte

A. B e ythien . . . . . $0,025-0,050$ 1905er Säfte

F. $L \ddot{u}$ h rigi ${ }^{1}$. . . . . . $0,055-0,066$

Küttner und $\left.\mathrm{C} l 1 \mathrm{rich} \mathrm{h}^{2}\right)$. $0,072-0,098$ selbstgepreßte Säfte

1) Ztechr. f. Unters. d. Nahr.- u. Genußm. 1906, 11, 444.

2) Ztschr. f. öffentl. Chom. 1806, 11, 1-10. 


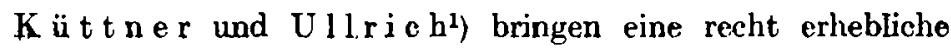
Anzahl von Zitronensaftuntersuchungen. Leider wird der Wert dieser Arbeit durch versehiedene Unriohtigkeiten bei der Berechnung, wie auch dadurch eingesahränkt, da $B$ das Untersuchungsmaterial für die auf Seite 208 erwähnten Analysen mehr oder weniger verdorben war. Auch im Hygienischen Institut zu Hamburg wurde ein ähnliches, nicht mehr einwandfreies Produkt ${ }^{2}$ ) derselben Bezugsquelle seinerzeit untersucht. Die selbsthergestellten Zitronensäfte der genannten Autoren haben ganz ähnliche Ergebnisse, wie die von K. Farnsteiner, A. Beythien und F. Lührig ergeben. Die Abwesenheit von Stickstoff wird von den Fabrikanten der Kunstprodukte oft mit ihrem sogen. Spezialverfahren entsehuldigt. In die Enge getrieben, wird dann meist angegeben, daß beim Filtrieren über Tierkohle der Stickstoff ganz oder zum Teil zurückgehalten sei. A. B e y th i e $\mathbf{n}^{3}$ ) stellte, um diese Behauptungen zu prüfen, verschiedene Versuche mit Tierkohle an und kam zu dem Ergebnis: daß zwar eine Behandlung mit Tierkohle den Stickstoffgehalt des Zitronensaftes wesentlich verringert, daß aber selbst vierstündiges Kochen mit großen Mengen 'Tierkohle nicht ausreicht, Produkte mit weniger als $30 \mathrm{mg}$ Stickstoff zu erzielen.

Die Größe des Extrakktrestes liegt meist zwischen $0,4-1,0 \mathrm{~g}$ in $100 \mathrm{ccm}$ Saft, während bei Kunsterzeugnissen, die keine auf Täuschung des Analytikers berechneten unbestimmbaren Zusätze erfahren haben, entweder annähernd der Wert 0 oder gar ein negativer Fxtraktrest gefunden wird. Es möge hier eingeschaltet werden, daß über den Begriff „Extraktrest" in der ZitronensaftLiteratur einige Unsicherheit herrscht. Unter „Extraktrest" ist im vorliegenden der Wert verstanden, welcher sich aus dem Extrakt durch Abzug von Zucker, Glyzerin, Säure, Mineralstoffen und an diese gebundene Zitronensäure ergibt.

Zur Beurteilung sind besonders die Werte für Mineralstoffe, Alkalität, Stickstoff und Extraktrest heranzuziehen. Mit dem Fortschreiten unserer Kenntnisse wurde die Zusammensetzung der Kunstprodukte derjenigen der Natursäfte immer ähnlicher; sie weist heute teilweise eine solche Uebereinstimmung mit den Naturprodukten auf, daß die Analyse vollständig normale Werte für Mineralstoffe, Alkalität und auch für Stickstoff liefern kann. In solchem Falle bleibt nur noch der Extraktrest allein zum Nachweis

1) Ztschr. f. öffentl. Chem. 1906, 11, 1-10.

2) Ztschr. f. Unters. d. Nahr.- u. Genußm. 1908, 6, 321-326.

3) Ztschr. f. Unters. d. Nahr.- u. Genußm. 1906, 15, I., 101. 
der Verfälschung zurück. Es empfiehlt sich dann, die Zusammensetzung der Mineralstoffe und Stickstoffverbindungen eingehend zu prüfen. Die qualitativen Reaktionen sind mit zur Beurteilung heranzuziehen; doch ist ihr Ausfall allein nicht maßgebend. Der erfahrene Chemiker wird es auch nicht unterlassen, die äußere Besohaffenheit der Säfte, Geruch und Geschmack bei der Beurteilung zu verwerten.

Die Gesichtspunkte, welche bei der Beurteilung der Konservierungsmittel in Frage kommen, sind unter anderen aus dem Gutachten der Königlichen Wissenschaftlichen Deputation für das Medizinalwesen ${ }^{1}$ ), betreffend die Verwendung von Salicylsijure zur Konservierung von Nahrungs- und Genußmitteln vom 8. Februar 1908, zu erseben. Als Konservierangsmittel wird in letzter Zejt außer Salicylsäure häufig Ameisensäure angetroffen.

Aus dem pharmakologischen Institut der Universitat Leipzig.

\section{Ueber Verbindungen des Wismuts mit einigen aliphatischen Oxysäuren.}

Von Dr. Hans Telle, Korps-Stabsapotheker beim XIX. Armeekorps.

(Eingegangen den 25. VII. 1908.)

Von den Verbindungen des Wismuts mit den wichtigsten Oxysäuren sind nur diejenigen der Weinsäure und Zitronensäure eingehender bearbeitet worden. Die Milchsäureverbindungen sind bisher nur sehr wenig umfangreich, und diejenigen der Apfelsäure meines Wissens nach überhaupt noch nicht beschrieben worden. Die Untersuchungsresultate der Weinsäureverbindungen des Wismuts widersprechen sich in vielen Punkten derart, daß es mir lohnend erschien, einerseits die bisherigen neueren Angaben und Resultate eingehender zu prüfen, andererseits die Verbindungen des Wismuts mit den Oxysäuren weiter zu erforschen.

Bevor ich auf die Verbindungen des Wismuts mit den Oxysäuren selbst eingehe, erlaube ich mir einige Untersuchungsresultate über das Wismuthydroxyd vorauszuschicken, wozu mich die An-

1) Ztschr. f. Unters. d. Nahr.- u. Genußm. 1908, 15, 440-445. 